\title{
Numerical models and experiment of air flow in a simulation box for optical wireless communications
}

\author{
Jan Latal ${ }^{1}$, Lukas Hajek${ }^{1}$, Marian Bojko², Jan Vitasek ${ }^{1}$, Petr Koudelka ${ }^{1}$, Stanislav Kepak ${ }^{1}$, Ales Vanderka ${ }^{1}$, Vladimir Vasinek ${ }^{1}$ \\ ${ }^{1}$ Department of Telecommunications, Faculty of Electrical Engineering and Computer Science, VSB-Technical University of Ostrava, \\ 17.listopadu 15, Ostrava-Poruba, 708 33, Czech Republic
}

${ }^{2}$ Department of Hydrodynamics and Hydraulic Equipment, Faculty of Mechanical Engineering, VSB-Technical University of Ostrava, 17.listopadu 15, Ostrava-Poruba, Czech Republic

\begin{abstract}
In this article, the authors focused on real measurements of mechanical turbulence generated by ventilators in the simulation box for Optical Wireless Communications. The mechanical turbulences disturb the optical beam that propagates along the central axis of the simulation box. The aim of authors is to show the effect of mechanical turbulence on optical beams at different heights in the simulation box. In the Ansys Fluent, we created numerical models which were then compared with real measurements. Authors compared the real and numerical models according to statistical methods.
\end{abstract}

\section{Introduction}

Today communication has become a necessity for people. It is necessary to be mobile and accessible on mobile devices that are connected to the Internet. This has resulted in increasing demands from internet service providers (ISPs) that must be able to cover large areas with sufficient signal levels. To satisfy this demand, ISPs are using all available communication systems including optical fiber-free connections. They are characterized by the fact that they do not need a licensed band for communication and are also easily installed on rooftops. Among the disadvantages that reduce the deployment of these systems is the need for line of sight between the transmitter and receiver and the influence of atmospheric phenomena.

For fiber-free optic communications the data and signal transmission uses an optical beam that is subject to atmospheric conditions, such as wind, rain, snow, turbulence, absorption, mist, fog, insects or birds, that can cause a decrease or loss of signal power level, or can change the spatial coherence of the optical beam. Before installing fiber-free links in a location, the implementation of a model behavior of the atmosphere and its influence on the fiber-free link is performed to reduce the overall degradation.

The aim of this article is to carry out a thorough analysis and real measurements of the air flow in a closed plexiglass box with dimensions of $250 \times 50 \times 50 \mathrm{~cm}$ and to create a numerical model in the ANSYS FLUENT 15 software, which is used for the simulation of fluid dynamics using the finite volume method (FVM) and for numerical modeling of the turbulent flow. Measured values will be compared to simulated values.

\section{Atmospheric turbulence}

The airflow in the atmosphere is divided into two kinds of flow: laminar flow and turbulent flow. During laminar flow the parallel motion of particles occurs. The particle motion is considered to be in layers that are mutually unmixed while friction among these layers is considered negligible.

However, for turbulent flow particle tracks are mixed. Particles do not obey laminar only motion, but also perform their own complex movement with irregular direction and speed in such a way that the flow can be considered to be stochastic and non-stationary. As the particles travel through different layers, these layers mix leading to the formation of vortices.

Atmospheric turbulence is divided into three types, namely thermal turbulence, turbulence and dynamic mechanical turbulence. In dynamic mechanical turbulence, a large wind shear occurs due to friction between the layers of air, each having different velocity and direction. In atmospheric optical links dynamic turbulence occurs only at altitudes above $4 \mathrm{~km}$ and could therefore affect satellite communication with the ground station for instance.

The airflow results in random fluctuations in the signal transmission quality, changing the power density incident on the detector. Random beam deflections lead to an increase in bit error rate or even to a complete loss of signal [1-6].

\footnotetext{
a Corresponding author: jan.latal@vsb.cz
} 


\subsection{The effects of turbulence on the optical beam}

Turbulence in the atmosphere are very unstable pockets of different sizes and densities unstable in time, continuously forming, changing and disappearing. They cause amplitude and phase fluctuations of coherent waves with time. Turbulence can be divided according to a relationship between the size of the light beam and the level of turbulence. All types of turbulence can impact transmission through the fluctuations in received power level at a frequency in the tens of $\mathrm{Hz}$.

\subsubsection{The diameter of the beam is smaller than the size of the turbulence}

If the optical beam diameter is smaller than the diameter of the turbulent vortex a deflection of the entire optical beam from its path takes place. The optical beam exits at a different angle from the turbulence as a refracted beam caused by the refraction index gradient of the turbulence. This may lead to a decrease in received power level to the point where only minimal optical power is detected.

\subsubsection{The diameter of the beam is the same size as turbulence}

If the optical beam has the same diameter as the turbulent vortex, turbulence acts as a lens which does not change the direction of the optical beam but increases its divergence. Additionally, this turbulence causes a constant change of attenuation and phase delay.

\subsubsection{The diameter of the beam is larger than the turbulence}

If the optical beam has a larger diameter than the turbulent vortex diameter, the beam is split into separate beams that exit the turbulent flow at different angles in addition, to the occurrence of intensity scattering and interference. The beams propagate along different paths resulting in a variation in the intensity of the optical beam called scintillation.

The relative variations in the optical intensity $\sigma_{I}^{2}$ are defined as:

$$
\sigma_{I}^{2}=\frac{\left\langle I^{2}\right\rangle-\langle I\rangle^{2}}{\langle I\rangle^{2}}=\frac{\left\langle I^{2}\right\rangle}{\langle I\rangle^{2}}-1
$$

where $\mathrm{I}$ is the optical intensity of the optical beam. If $\sigma_{I}^{2}<<1$, we can use Rytovov approximation which works even with the structural parameter of the refractive index $C_{n}^{2}$ :

$$
\sigma_{I}^{2}=K \cdot C_{n}^{2} \cdot k^{7 / 6} \cdot L^{11 / 6}
$$

where $K$ represents a constant for a plane wave equal to 1.23 and for a spherical wave equal to $0.5, k$ is the wave number and $L$ is the distance between the transmitter and receiver.
If the relative variations change of the refractive index of is less than $1 \sigma_{I}^{2}<1$ it is a weak turbulence. If this condition is not met, it is a strong turbulence.

Scintillation can be eliminated by using multiple transmitters or using large area receivers. The amplitude and frequency of the scintillation depends on the cell size compared to the size of the light beam. The amplitude and frequency of the scintillation increases with the increasing frequency of light.

For a plane waves in a low turbulence environment and for a specific receiver the scintillation variance can be expressed by the following formula:

$$
\alpha_{\text {sin }}=2 \sqrt{23,17 \cdot\left(\frac{2 \pi}{\lambda} 10^{9}\right)^{\frac{7}{6}} \cdot C_{n}^{2} \cdot L^{\frac{11}{6}}}[d B],
$$

where the individual variables can be described as follows $\lambda$ transmission wavelength [nm], $L$ transmission channel distance $[\mathrm{m}], C_{n}{ }^{2}$ structural parameter of the refractive index $\left[\mathrm{m}^{-2 / 3}\right]$.

According to Andrews, the optical intensity variation equation is expanded to include the diameter of the receiving lens $D_{R X A}$. This idea is based on the assumption that turbulence is not absorbing optical power as in the case of absorption, but merely diverting the direction of the beam. These diverted beams are then focussed on the lens surface of the detector.

$$
\begin{aligned}
& \sigma_{I}^{2}\left(D_{R X A}\right)=\exp \left(\frac{0,49 \cdot \beta^{2}}{\left(1+0,18 \cdot d^{2}+0,56 \cdot \beta^{12 / 5}\right)^{7 / 5}}+\right. \\
& \left.+\frac{0,51 \cdot \beta\left(1+0,69 \cdot \beta^{12 / 5}\right)^{-5 / 6}}{\left(1+0,90 \cdot d^{2}+0,62 \cdot d^{2} \cdot \beta^{12 / 5}\right)^{7 / 5}}-1\right)
\end{aligned}
$$

Coefficients $\beta$ and $d$ are determined as:

$$
\beta=0,5 \cdot C_{n}^{2} \cdot k^{7 / 5} \cdot L^{11 / 6}, d=\sqrt{\frac{2 \pi}{4 \lambda L}} D_{R X A}
$$

Attenuation expressed using the Andrews approximation and can be written as:

$$
\alpha_{\text {turbulence }}=10 \log \left|1-\sqrt{\sigma_{\mathrm{I}}^{2}\left(\mathrm{D}_{\mathrm{RXA}}\right)}\right|
$$

The Andrews expression of attenuation due to the turbulent atmosphere envisages the lowest possible value of attenuation. Another method which is focused on the determination of turbulent attenuation is called the method of available power. It is used for the sources with a Gaussian distribution of the optical intensity in the plane perpendicular to the direction of propagation [1-6].

\section{Characteristics of the mathematical model for fluid flow}

The flow of fluids (gases and liquids) is divided into laminar and turbulent flow. To determine the nature of flow is used dimensionless parameter called the Reynolds number: 


$$
\operatorname{Re}=\frac{\mathrm{v} \cdot \mathrm{d}_{\mathrm{h}}}{v},
$$

where $\left(\mathrm{d}_{\mathrm{h}}\right)[\mathrm{m}]$ describes the characteristic diameter equal to the diameter of the inlet hole, $(\mathrm{v})\left[\mathrm{m} \cdot \mathrm{s}^{-1}\right]$ velocity of air and $(v)\left[\mathrm{m}^{2} \cdot \mathrm{s}^{-1}\right]$ kinematic viscosity of flowing media. Type of flow can be determined from the size of the Reynolds number. In the determination of the boundaries between laminar and turbulent flow is discovered many values which may vary by pressure, altitude, or temperature. The approximate values of $2320<\operatorname{Re}<4000$, where $\operatorname{Re}<2320$ is the laminar flow and at $\operatorname{Re}>4000$ is the turbulent flow are taken into consideration. The area between them is known as the transition region [10]. Laminar flow is considered to be a fluid flow of such a viscosity that the streamlines does not mix and are therefore parallel to the propagation direction of the medium. This type of flow can be observed for example in liquids at low speeds and high viscosity. On the contrary, turbulent flow shows chaotic changes (fluctuations) in the case of temperature, pressure, speed and direction of flow [5, 8]. The modelling of turbulent flow can be made using many methods made possible by the computing power of today's computers. The basic techniques include simulations of large eddies (LES) or direct numerical simulation (DNS). However, the DNS method becomes technically unfeasible because of the growing complexity of models, size of computational net and Reynolds number. One of the most used methods today is the Reynolds averaging of the Navier-Stokes equations (RANS). This method is based on the time-averaged values of turbulent flow and time-averaging procedure at balance equations $[5,9]$.

$$
\begin{gathered}
\zeta=\bar{\zeta}+\zeta^{\prime} \\
\bar{\zeta}=\frac{1}{\mathrm{~T}} \int_{0}^{\mathrm{T}} \zeta \mathrm{dt}, \bar{\zeta}^{\prime}=0
\end{gathered}
$$

Individual immediate values of the turbulent flow $(\zeta)$ are divided into the sum of the median value $(\bar{\zeta})$ and fluctuating value $\left(\zeta^{\prime}\right)$.

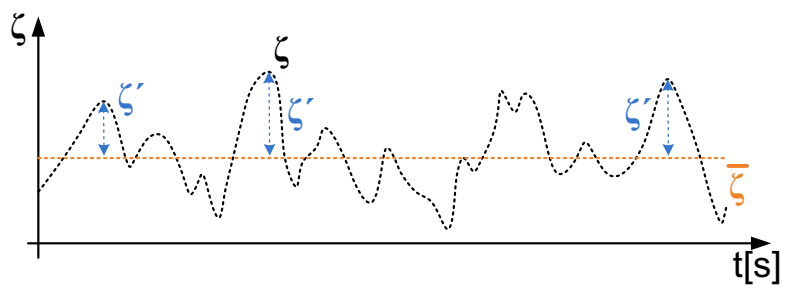

Figure 1. Mean, fluctuation, immediate value [7].

The median value is the arithmetic average in a specific period $T$ and the average value of fluctuations is equal to zero (see equation (9)). Based on equation (8) and (9) the equation of continuity (10) and N-S equation (11) of incompressible, time-independent flow can be defined into the form:

$$
\begin{gathered}
\frac{\partial \overline{\mathrm{u}}_{\mathrm{j}}}{\partial \mathrm{x}_{\mathrm{j}}}=0, \\
\frac{\partial\left(\bar{u}_{i} \bar{u}_{j}\right)}{\partial x_{j}}+\frac{\partial\left(\overline{u_{i}^{\prime} u_{j}^{\prime}}\right)}{\partial x_{j}}=-\frac{1}{\rho} \frac{\partial(\bar{p})}{\partial x_{i}}+v \frac{\partial^{2}\left(\overline{u_{i}}\right)}{\partial x_{j}^{2}}+\overline{f_{i}}
\end{gathered}
$$

After adjustment of the N-S equations, the tensor of Reynolds (turbulent) stress arises in the link (11), the tensor must be numerically modelled. In order to limit the computing demand on the calculation of the turbulent flow, the Boussinesq hypothesis was introduced. The hypothesis uses the Newtonian relation to replace the tensor of nine shear stresses with one quantity of the same unit as the dynamic viscosity. The result of the Boussinesq hypothesis is the modified N-S equation for noncompressible, time-independent flow:

$$
\frac{\partial\left(\bar{u}_{i} \bar{u}_{j}\right)}{\partial x_{j}}=-\frac{1}{\rho} \frac{\partial(\bar{p})}{\partial x_{i}}+v_{e f f} \frac{\partial^{2}\left(\bar{u}_{i}\right)}{\partial x_{j}^{2}}+\overline{f_{i}} .
$$

The Boussinesq hypothesis is the basis of numerical models that have lower computational requirements in calculating the turbulent viscosity. The division into zero, one and two-linear equation models is represented. The Zero-linear equation model refers to the fact that apart from the equation of conservation of energy, momentum and mass no transport equations are needed. Next is the single linear equation model, which uses a transport equation for modeling the turbulent kinetic energy that is a function of the turbulent viscosity. However it is necessary to specify the turbulent rule that is dependent on the qualities of the flow. The zero-linear and onelinear equation models are called incomplete models because it is necessary to know the character of the flow during the calculation of these models. When modeling the turbulent flow, the two-linear equation models $\mathrm{k}-\omega$ a $\mathrm{k}-\varepsilon$ [2] are defined the most often. The models which are used in this work are the two-linear equation models: $\mathrm{k}-\varepsilon$ Standard, k- $\varepsilon$ RNG, k- $\varepsilon$ Realizable, k- $\omega$ Standard and k- $\omega$ SST.

\section{Simulation of mechanical turbulence}

CFD simulations of air flow in the box were made using the ANSYS Fluent 15 software. To create a numerical model, the geometric model must be made first. The computational grid with the appropriately set boundary conditions is then applied to the geometric model. The turbulent models which are used in this work are the two-linear equation models k- $\varepsilon$ (Standard, RNG, Realizable) and k- $\omega$ (Standard and SST). For solution of turbulent flow are many models. Models are suitable for different characters of flow and different conditions of flow. In this article we focus on experimental measurements and mathematical modelling. Therefore, the use of powerful CFD model of turbulence will be the content follow research turbulent flow. Some short 
information about characteristics of others turbulent models. DES models have been specifically designed to address high Reynolds number wall bounded flows, where the cost of a near-wall resolving Large Eddy Simulation would be prohibitive. The difference with the LES model is that it relies only on the required RANS resolution in the boundary layers. The application of DES, however, may still require significant CPU resources and therefore, as a general guideline, it is recommended that the conventional turbulence models employing the Reynolds-averaged approach be used for most practical calculations. Resolving only the large eddies allows one to use much coarser mesh and larger time-step sizes in LES than in DNS. However, LES still requires substantially finer meshes than those typically used for RANS calculations. In addition, LES has to be run for a sufficiently long flow-time to obtain stable statistics of the flow being modeled. As a result, the computational cost involved with LES is normally orders of magnitudes higher than that for steady RANS calculations in terms of memory (RAM) and CPU time. Therefore, high-performance computing (for example, parallel computing) is a necessity for LES, especially for industrial applications. Next turbulent models are ScaleAdaptive Simulation (SAS), Embedded Large Eddy Simulation (ELES), Reynolds Stress Model (RSM), more information [11], [12]. For application these models will be necessary to ensure performance computing serve.

\subsection{CFD model for simulation in plexiglass box}

As stated previously, the actual measurement was carried out in plexiglass boxes with dimensions of $2.5 \times$ $0.5 \times 0.5 \mathrm{~m}$. In ANSYS Fluent 15 the geometric model of the box was created. Then the boundary conditions derived from real measurements were applied to the model. Using the Extrude method a $0.5 \mathrm{~m}$ height box was made. Four inlet and outlet holes with the shape of a circle with $11 \mathrm{~cm}$ diameter were created in the model. Because fan comprises of a rotor and a stator it was necessary to create another circle with $5.4 \mathrm{~cm}$ diameter inlets these circles, which then represents the stator. These prepared inlets to the plexiglass box were modeled like in the real box. The geometric model of the box can be seen in Figure 2.

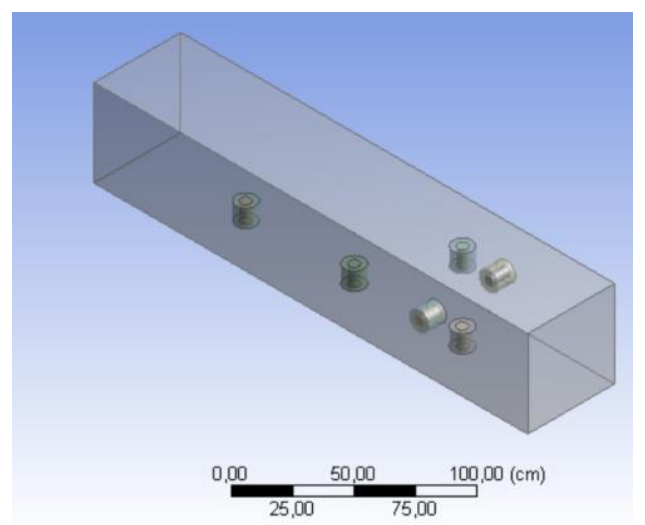

Figure 2. Geometric model of the box.
After the creation of the geometric model and computational network the boundary conditions were set. These settings were based on the real measurement data. The velocity for individual fans and outlets were measured, as shown in Figure 3.

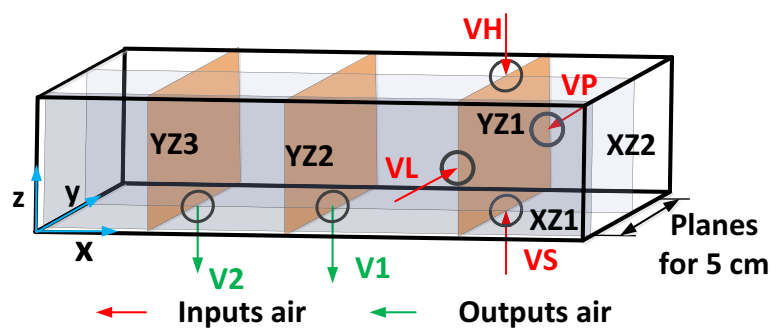

Figure 3. Nine measured planes for $5 \mathrm{~cm}$ in the plexiglass model box.

Whirling flow behavior of the air media was generated using the inlet fan rotor blades mounted on the bottom side at the center hole. A SUNON A2123-HBT fan was used. The average measured velocity at the individual inlets and outlets are listed in Table 1. The most essential part of the boundary conditions setting has been given to the inlet. It was important to stick with the same parameters with the actual model as closely as possible. After the setting of these values in the simulation, it was found that the average velocity at the inlets was around $11 \mathrm{~m} \cdot \mathrm{s}^{-1}$ and $10 \mathrm{~m} \cdot \mathrm{s}^{-1}$ at the outlets. This could be mainly due to the fact that the simulation is actually an expression of the ideal state, which does not calculate fan blades that must be modeled and inserted into a simulation separately. By gradually reducing the velocity it was discovered that it is best to count with the inlet velocity at about $1.7 \mathrm{~m} \cdot \mathrm{s}^{-1}$ lower. After using this reduction in speed method, it was found that the average velocity reached values that were read during real measurements at the inlet and outlets.

Table 1. Measured velocity at the box inlets and outlets.

\begin{tabular}{|c|c|}
\hline Box inlets and outlets & $\begin{array}{c}\text { Velocity } \\
{\left[\mathbf{m} \cdot \mathbf{s}^{\mathbf{1}}\right]}\end{array}$ \\
\hline Left inlet & 7,00 \\
\hline Bottom inlet & 6,75 \\
\hline Right inlet & 6,65 \\
\hline Top inlet & 6,25 \\
\hline Outlet & 8,00 \\
\hline Outlet & 8,00 \\
\hline
\end{tabular}

Subsequently, the mass flow rate was calculated by the following formula:

$$
Q_{m}=S \cdot \mathrm{v} \cdot \rho=\pi \cdot r^{2} \cdot \mathrm{v} \cdot \rho,
$$

where $S$ represents the content of the inlet area, $v$ is velocity, and $\rho$ is the air density. Since we know that the fans are formed by two parts the rotor and stator, it is 
necessary to obtain an area value which defines the area of flow. Therefore, the surface of the rotor and stator are subtracted to obtain the total inlet area. The parameter of turbulent intensity and the hydraulic diameter at the inlet can be set for the boundary conditions within the simulation. The hydraulic diameter is defined as a difference between the internal and external diameter of the box inlet and outlet holes. The turbulent intensity is derived from Reynolds numbers [7-9] and can be calculated using the following equation:

$$
I=0.16 \cdot(\mathrm{Re})^{-\frac{1}{8}},[\%]
$$

To make the turbulent intensity calculation for the right intake fan it was first necessary to calculate the inlet area contents:

$$
\begin{gathered}
\mathrm{S}=\mathrm{S}_{1}-\mathrm{S}_{2} \\
\mathrm{~S}_{1}=\pi \cdot \mathrm{r}^{2}=3.14 \cdot 5.52=94.98 \mathrm{~cm}^{2} \\
\mathrm{~S}_{2}=\pi \cdot \mathrm{r}^{2}=3.14 \cdot 2.72=22.8906 \mathrm{~cm}^{2} \\
\mathrm{~S}_{\mathrm{cel}}=94.985-22.8906=72.0944 \mathrm{~cm}^{2}=0.0072 \mathrm{~m}^{2}
\end{gathered}
$$

Air density was $1,184 \mathrm{~kg} \cdot \mathrm{m}^{-3}$ at a temperature of $25{ }^{\circ} \mathrm{C}$. Based on these values, we can calculate the mass flow rate defined as:

$$
\begin{aligned}
& Q_{m}=S \cdot \mathrm{v} \cdot \rho=0.0072 \cdot 5.3 \cdot 1.184= \\
& =0.04518144 \mathrm{~kg} \cdot \mathrm{s}^{-1}
\end{aligned}
$$

The hydraulic diameter is given by:

$$
\begin{aligned}
& \mathrm{d}_{\mathrm{h}}=\text { Average } S_{1}-\text { Average } S_{2}=11-5.4= \\
& =5.6 \mathrm{~cm}=0.056 \mathrm{~m}
\end{aligned}
$$

Then using a formula to calculate the Reynolds number, we compute the value of $R_{e}=1658$. This value is substituted into the formula for the turbulent intensity calculation, leading to a value of $\mathrm{I}=4.7 \%$ for the left intake fan. The following table 2 lists the overview of the calculations for individual inlets.

Table 2. Calculated values for mass flow rate, turbulent intensity and hydraulic diameter.

\begin{tabular}{|c|c|c|}
\hline \multirow{2}{*}{$\begin{array}{c}\text { Fan type } \\
\text { VPight) }\end{array}$} & Quantity & Value \\
\cline { 2 - 3 } & $\mathrm{Qm}_{\mathrm{m}}\left[\mathrm{kg} \cdot \mathrm{s}^{-1}\right]$ & 0.04219776 \\
\cline { 2 - 3 } & $\mathrm{I}[\%]$ & 4.7 \\
\hline \multirow{2}{*}{$\begin{array}{c}\text { VS } \\
\text { (bottom) }\end{array}$} & $\mathrm{Qm}_{\mathrm{m}}\left[\mathrm{kg} \cdot \mathrm{s}^{-1}\right]$ & 0.04305024 \\
\cline { 2 - 3 } & $\mathrm{I}[\%]$ & 4.7 \\
\hline
\end{tabular}

\begin{tabular}{|c|c|c|}
\hline & $\mathrm{d}_{\mathrm{h}}[\mathrm{m}]$ & 0.056 \\
\hline \multirow{3}{*}{ VH (top) } & $\mathrm{Q} \mathrm{m}\left[\mathrm{kg} \cdot \mathrm{s}^{-1}\right]$ & 0.03878784 \\
\cline { 2 - 3 } & $\mathrm{I}[\%]$ & 4.8 \\
\cline { 2 - 3 } & $\mathrm{d}_{\mathrm{h}}[\mathrm{m}]$ & 0.056 \\
\hline
\end{tabular}

The pressure-outlet boundary condition at the inlets and mass-flow-rate-inlet boundary condition at the outlets was used in the simulation.

\section{The real measurements of mechanical turbulence}

When measuring mechanical turbulence, four fans were mounted on the right side of each wall and two bottom outlets were exposed. Measurements were performed using an anemometer (type Almemo 2290-2/3) capable of real-time air velocity measurement. The box was imaginarily divided into 9 vertical planes spaced apart by $5 \mathrm{~cm}$, see Figure 3. In each plane 459 notional points were spaced $5 \mathrm{~cm}$ apart resulting in 4131 measured points. Because at every point the air flows from different directions, the measurement has to be made in three axes $\mathrm{x}, \mathrm{y}$ and $\mathrm{z}$. Overall we get 12393 measurements leading to the air velocity values. In this way, the whole box was measured. To obtain the actual value of the velocity at that point, we have to use following formula:

$\|\mathrm{v}\|=\sqrt{\mathrm{v}_{\mathrm{x}}^{2}+\mathrm{v}_{\mathrm{y}}^{2}+\mathrm{v}_{\mathrm{z}}^{2}}$

where $v$ is the velocity magnitude at a given point and $v_{x}, v_{y}, v_{z}$ represents the measured values in different mutually perpendicular axes. These calculated velocity values were then processed and plotted in graphs using Matlab R2014a. In Figure 4 the air velocity in XZ1 plane can be seen. This plane is $5 \mathrm{~cm}$ distant from the beginning of the box. It can be seen that the largest velocity occurs in the area of air inlet VL, see Figure 3, with successively decreasing air velocity occuring in the direction of V1 and V2 outlets.

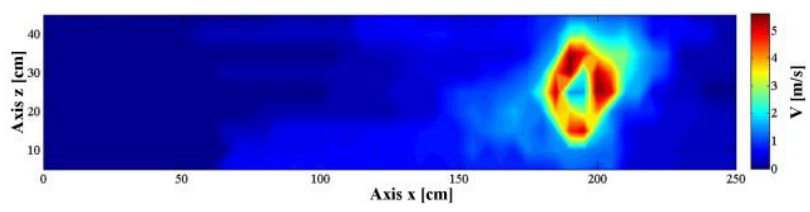

Figure 4. The air velocity in XZ1 plane

In the XZ2 plane which is depicted in Figure 5, the largest air velocity is apparent in the area of intake fans $\mathrm{VS}$ and $\mathrm{VH}$. The measured velocity averaged at $7 \mathrm{~m} \cdot \mathrm{s}^{-1}$ and gradually declined. A smaller portion of the energy is located in the area of the outlets V1 and V2. This plane goes exactly through the center of the box which is also $25 \mathrm{~cm}$ away from the outer wall. 


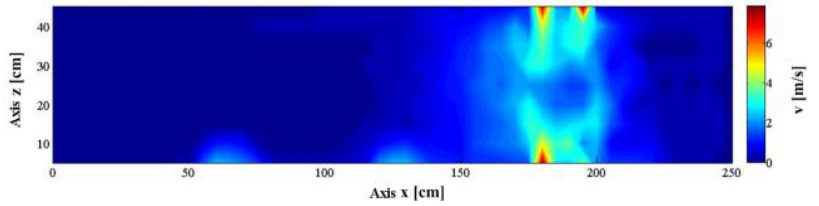

Figure 5. The air velocity in XZ2 plane.

Figure 6 shows three planes, the first two planes YZ3 and YZ2 pass through the middle of the V1 and V2 outlets and YZ1 plane intersects the center of the fan inlets VL, $\mathrm{VS}, \mathrm{VP}$ and VH. Visible difference in air velocity are between both outlets, where the V2 outlet shows larger decrease (up to $2.4 \mathrm{~m} \cdot \mathrm{s}^{-1}$ ) than the $\mathrm{V} 1$ outlet where maximum air velocity was $2.8 \mathrm{~m} \cdot \mathrm{s}^{-1}$. In the last plane YZ1, the air pumping from the fans is apparent and also the pumped air profile formed by the two turbulent lobes. It is caused due to the fact that the fan is formed by a rotor and stator whereby air can only pass through the rotor blades.

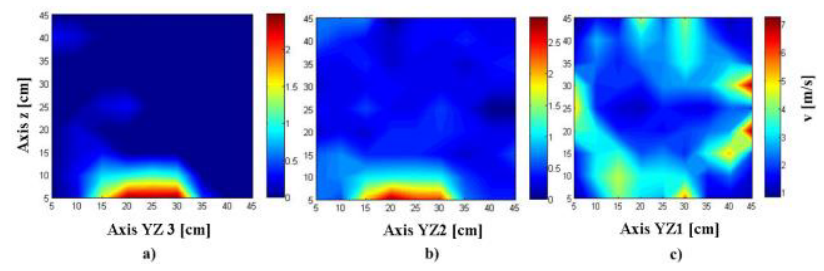

Figure 6. The air velocity: a) in YZ3 plane, b) in YZ2 plane, c) in YZ1 plane.

Individual velocity profiles at the fan inlets and outlets can be seen in Fig. 7 and 8. The maximum fan air velocity was determined to be around $10 \mathrm{~m} \cdot \mathrm{s}^{-1}$, the average being however around $7 \mathrm{~m} \cdot \mathrm{s}^{-1}$. This average value was then set in simulation software. The maximum air velocity at the outlets was around $8 \mathrm{~m} \cdot \mathrm{s}^{-1}$.
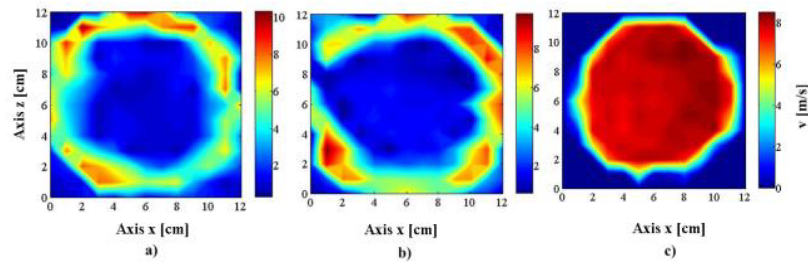

Figure 7. The air velocity a) VL fan, b) VS fan, c) V1 outlet.
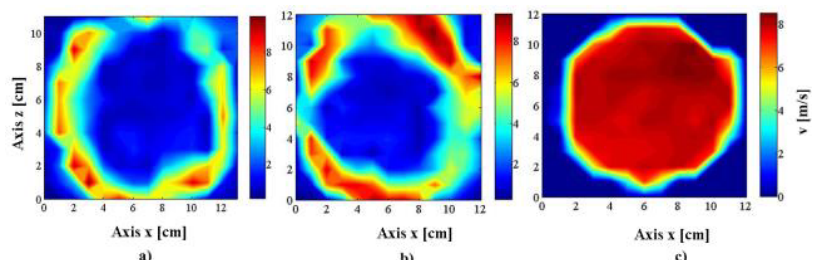

Figure 8. The air velocity a) VP fan, b) VH fan, c) V2 outlet.

\section{Statistical comparisons of the numerical models}

To compare different numerical models, three different methods were chosen. The first method used an averaging of the air velocity in individual planes. Another method used in the numerical model comparison used MSE, which is the root mean square error, and the last method used the NSE (Nash-Sutcliffe Efficiency). In Table 3 we can see the comparison of numerical models with real measured data by averaging the velocity in defined planes (see Figure 3). The highlighted models are the ones that most closely match the measured data. From Table 3 it is apparent that the $\mathrm{k}-\varepsilon$ RNG numerical model is the most similar to the measured values.

Table 3. Velocity averages comparison in defined planes. Velocity are in $\mathrm{m} \cdot \mathrm{s}^{-1}$.

\begin{tabular}{|c|c|c|c|c|c|c|}
\hline $\begin{array}{c}\text { Inlets } \\
/ \\
\text { Outle } \\
\text { ts }\end{array}$ & $\begin{array}{c}\text { Meas } \\
\text { ured } \\
\text { values }\end{array}$ & $\begin{array}{c}k-\varepsilon \\
\text { Stand } \\
\text { ard }\end{array}$ & $\begin{array}{l}k-\varepsilon \\
\text { RNG }\end{array}$ & $\begin{array}{c}\mathbf{k}-\varepsilon \\
\text { Realiz } \\
\text { able }\end{array}$ & $\begin{array}{c}\mathbf{k}-\omega \\
\text { Stand } \\
\text { ard }\end{array}$ & $\begin{array}{c}k-\omega \\
\text { SST }\end{array}$ \\
\hline VL & 7 & 7.28 & 7.28 & 7.28 & 7.28 & 7.28 \\
\hline VS & 6.75 & 6.94 & 6.94 & 6.94 & 6.94 & 6.94 \\
\hline VP & 6.65 & 6.8 & 6.8 & 6.8 & 6.8 & 6.8 \\
\hline VH & 6.25 & 6.25 & 6.25 & 6.25 & 6.25 & 6.25 \\
\hline V1 & 8 & 7.74 & 7.99 & 7.94 & 7.75 & 7.97 \\
\hline $\mathrm{V} 2$ & 8 & 7.8 & 8.5 & 8 & 7.95 & 8 \\
\hline \multicolumn{7}{|c|}{ Plane } \\
\hline XZ1 & 0.60 & 0.462 & 0.58 & 0.433 & 0.613 & 0.058 \\
\hline
\end{tabular}

As part of the evaluation of the numerical model that most closely resembles the measurements, $\mathrm{k}-\varepsilon \mathrm{RNG}$ model was chosen (see Figure 9). The simulation results in individual cross sections or planes are shown in the following figures. The numerical model $\mathrm{k}-\varepsilon \mathrm{RNG}$ is an extension of $\mathrm{k}-\varepsilon$ Standard model, when it is apparent that $\mathrm{k}-\varepsilon \mathrm{RNG}$ in greater extent calculates the turbulent flow at inlets and outlets, see Figure 10 and at the individual planes (see Figure 11) of the simulated box. 


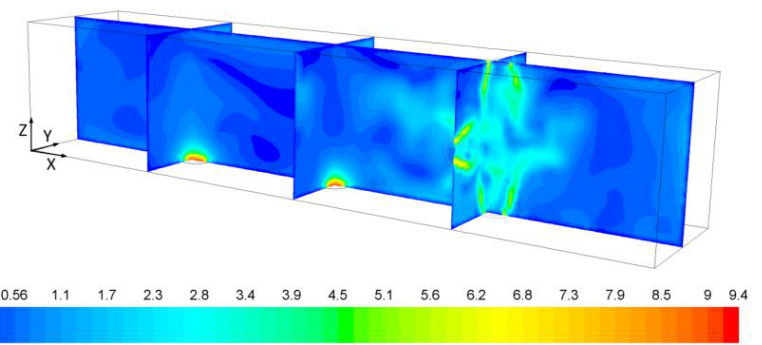

Figure 9. The velocity for different planes calculated using k$\varepsilon$ RNG model.

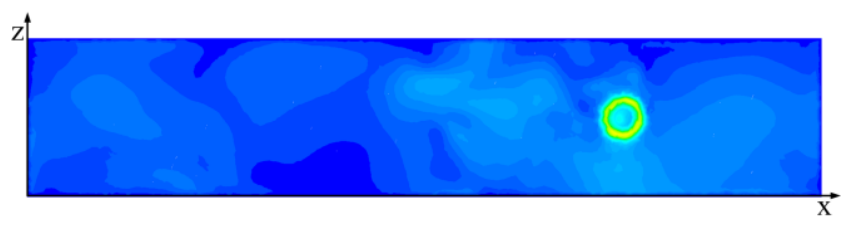

Figure 10. Section view of the velocity in $X Z 1$ plane in the simulated box and for $\mathrm{k}-\varepsilon \mathrm{RNG}$ model.

Figure 10 depicts the air velocity around the simulated box intake fan in XZ1 plane and calculated using $\mathrm{k}-\varepsilon$ RNG model. In XZ2 plane, the same simulation can be seen from a different perspective (Figure 11). The individual planes YZ1, YZ2 and YZ3 represent the simulated box cross sections according to Figure 3.
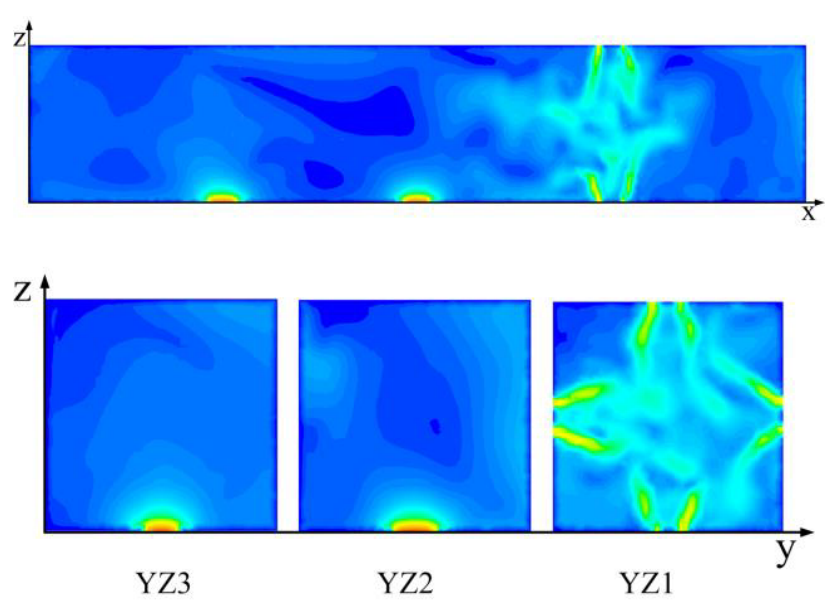

Figure 11. Section view of the velocity in XZ2, YZ1, YZ2 and YZ3 planes in the simulated box and for $\mathrm{k}-\varepsilon$ RNG model.

\subsection{XZ1 plane evaluation}

To determine the influence of turbulence on the optical beam, the XZ1 sectional evaluation was selected. The MSE and NSE methods were used to calculate the individual coefficients on selected layers, see Tables 4 and 5 .
Table 4. Calculated coefficients for MSE method.

\begin{tabular}{|c|c|c|c|c|c|}
\hline \multicolumn{6}{|c|}{ Models } \\
\hline cm & $\begin{array}{c}\mathbf{k}-\varepsilon \\
\text { Standar } \\
\mathbf{d}\end{array}$ & $\begin{array}{l}k-\varepsilon \\
\text { RNG }\end{array}$ & $\begin{array}{c}\mathrm{k}-\varepsilon \\
\text { Realiz } \\
\text { able }\end{array}$ & $\begin{array}{c}\mathbf{k}-\omega \\
\text { Standa } \\
\mathbf{r d}\end{array}$ & $\begin{array}{c}\mathbf{k}-\omega \\
\text { SST }\end{array}$ \\
\hline 5 & 0.140 & 0.138 & 0.063 & 0.053 & 0.143 \\
\hline 10 & 0.222 & 0.120 & 0.134 & 0.072 & 0.143 \\
\hline 15 & 1.085 & 0.737 & 0.831 & 0.550 & 0.831 \\
\hline 20 & 0.679 & 0.695 & 0.634 & 0.549 & 0.534 \\
\hline 25 & 1.402 & 1.190 & 1.334 & 1.256 & 1.256 \\
\hline 30 & 1.025 & 1.052 & 0.975 & 0.888 & 1.067 \\
\hline 35 & 1.265 & 1.412 & 1.350 & 1.277 & 1.355 \\
\hline 40 & 0.317 & 0.387 & 0.320 & 0.355 & 0.290 \\
\hline 45 & 0.134 & 0.188 & 0.077 & 0.069 & 0.192 \\
\hline
\end{tabular}

Table 5. Calculated coefficients for NSE method.

\begin{tabular}{|c|c|c|c|c|c|}
\hline \multicolumn{5}{|c|}{ Models } \\
\hline $\mathbf{c m}$ & $\begin{array}{c}\mathbf{k}-\mathbf{\varepsilon} \\
\text { Standar } \\
\mathbf{d}\end{array}$ & $\begin{array}{c}\mathbf{k}-\boldsymbol{\varepsilon} \\
\mathbf{R N G}\end{array}$ & $\begin{array}{c}\mathbf{k}-\boldsymbol{\varepsilon} \\
\text { Realiz } \\
\mathbf{a b l e}\end{array}$ & $\begin{array}{c}\mathbf{k}-\mathbf{\omega} \\
\text { Standa } \\
\mathbf{r d}\end{array}$ & $\begin{array}{c}\mathbf{k}-\mathbf{\omega} \\
\text { SST }\end{array}$ \\
\hline $\mathbf{5}$ & -0.125 & -0.111 & 0.497 & 0.574 & -0.149 \\
\hline $\mathbf{1 0}$ & 0.291 & 0.359 & 0.289 & 0.618 & 0.239 \\
\hline $\mathbf{1 5}$ & -0.136 & 0.228 & 0.130 & 0.423 & 0.129 \\
\hline $\mathbf{2 0}$ & 0.295 & 0.278 & 0.342 & 0.430 & 0.445 \\
\hline
\end{tabular}




\begin{tabular}{|c|c|c|c|c|c|}
\hline $\mathbf{2 5}$ & 0.152 & 0.280 & 0.194 & 0.241 & 0.241 \\
\hline $\mathbf{3 0}$ & 0.408 & 0.393 & 0.437 & 0.488 & 0.385 \\
\hline $\mathbf{3 5}$ & 0.101 & 0 & 0.040 & 0.092 & 0.036 \\
\hline $\mathbf{4 0}$ & 0.118 & -0.080 & 0.108 & 0.010 & 0.192 \\
\hline $\mathbf{4 5}$ & 0.051 & -0.333 & 0.456 & 0.508 & 0.460 \\
\hline
\end{tabular}

The highlighted areas show the greatest agreement with the measured values. The XZ1 plane closest to the box wall $(5 \mathrm{~cm})$ was simulated best using the $\mathrm{k}-\omega$ Standard model. The simulated vs real measured velocity values of individual models at $25 \mathrm{~cm}$ level can be seen in Figure 12. The blue line represents the measured values while the dashed line the most realistic model. The measured values of velocity were up to $5.5 \mathrm{~m} \cdot \mathrm{s}^{-1}$ and the best approximation to that value was using the $\mathrm{k}-\varepsilon \mathrm{RNG}$ model (solid red line in Figure 12).

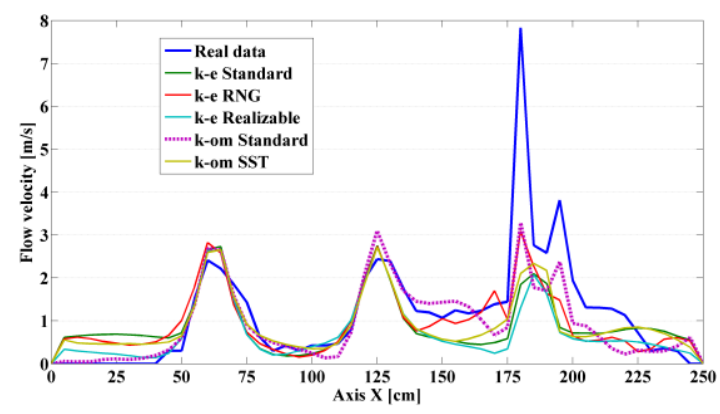

Figure 12. Model comparison at $25 \mathrm{~cm}$ level.

\section{Conclusion}

In this article the team of authors have focused on the modelling of air flow in a simulation box used for modelling atmospheric conditions. From the measured data the realistic model was determined using different types of numerical models in the ANSYS Fluent 15 software. These models were compared and evaluated. Based on the NSE and MSE statistical methods, the numerical model that most closely corresponded to the actual measurements was chosen. The selected model can therefore be used to the model the air flow in the box.

\section{Acknowledgement}

The research described in this article was undertaken thanks to the active support of the Ministry of Education, Youth and Sports of the Czech Republic through grant project no. CZ.1.07/2.3.00/20.0217 within the frame of the operation programme Education for competitiveness financed by the European Structural Funds and from the state budget of the Czech Republic. This article was also supported by project Technology Agency of the Czech Republic TA03020439 and TA04021263. The research has been partially supported by the projects no. SP2015/130, SP2015/182 and VI20152020008.

\section{References}

1. T. David, J. Latal, F. Hanacek, P. Koudelka, J. Vitasek, J., P. Siska, J. Skapa, V. Vasinek, Advances in Electrical and Electronic Engineering 9, 4 (2011)

2. O. Bouchet, J.C. Ricklin. Wireless optical telecommunications: principles and advances, John Wiley, Hoboken (2012)

3. R. Ramirez-Iniguez, S.M. Idrus, Z. Sun. Optical wireless communications: IR for wireless connectivity. CRC Press, Boca Raton (2008)

4. A. Vanderka, L. Hajek, J. Latal, J. Vitasek, P. Koudelka, Advances Electrical and Electronic Engineering, 12, 6 (2014)

5. L. Hajek, J. Latal, M. Bojko, R. Poboril, P. Koudelka, J. Vitasek, P. Siska, V. Vasinek. EPJ Web of Conferences 67, 02032 (2014)

6. A.K. Majumdar, J.C. Ricklin. Free Space Laser Communications Principles and Advances, Springer ISBN 978-0-387-28652-5 (2008)

7. M. Kozubkova, Modeling of fluid flow: FLUENT, CFX. Ostrava: Technical University of Ostrava, (2008)

8. T. Blejchar, Turbulence - Flow modeling - CFX. Ostrava: Technical University of Ostrava, ISBN 97880-248-2606-6 (2010)

9. M. Kozubkova, S. Drabkova. Numerical modeling of flow: FLUENT I. Ostrava: Technical University of Ostrava, ISBN 80-248-0525-1 (2003)

10. M. Kozubkova, T. Blejchar, M. Bojko, Modelling of heat, mass transfer and momentum. Ostrava: Technical University of Ostrava, ISBN 978-80-2482491-8 (2013)

11. FLUENT: Fluent 15.0 - ANSYS FLUENT, ANSYS, Inc., (2013)

12. D. C. Wilcox. Turbulence Modeling for CFD. D C W Industries. 522p. (2006) 